\title{
Der Lucius-Burckhardt-Platz in Kassel \\ Eine Geschichte über den Kampf um Legitimität und Deutungshoheit bei der Neugestaltung des Campus der Universität Kassel
}

Sieben Textfresser*innen von der Initiative „Lucius-Burckhardt-Platz bleibt!“

Die Uni wächst. Mitten in der Stadt. In Kassel. Lucius Burckhardt (†2003) war auch mal da. Aber das ist schon lange her. Zur großen Kunstausstellung (Documenta 14, Kassel \& Athen 2017) soll er wieder kommen, aber auf seinem Platz ist kein Platz für ihn. Vielleicht bleibt ein Grabstein für seine Ideale oder es wird ihm einfach ein neuer schöner Platz gebaut. Nur: Warum sollte der dann seinen Namen tragen?

Wir sind ein temporäres Autor*innenkollektiv mit sieben Textfresser*innen aus Kassel. Einige von uns setzten sich seit 2011 für den Erhalt des Lucius-Burckhardt-Platzes am Rande der Universität ein. Als Mitglieder der Initiative „Lucius-Burckhardt-Platz bleibt!“ erzählen wir euch hier von unserem Versuch, die Zerstörung und autoritäre Überplanung dieses Quartiersplatzes zu verhindern. Es ist die Geschichte unseres Widerstandes gegen eine Planung, die Teilhabe als Feigenblatt auf der Ebene von institutionellen Gremien und Ausschüssen proklamiert. Gegen eine Planung, die gewachsene soziale und räumliche Strukturen negiert und die Wünsche der Nutzer*innen mit Absicht überhört. Wir werden Hintergründe und Mechanismen dieser politischen Planungspraxis an der Universität Kassel aufzeigen und über unsere Erfolge, unsere Kompromisse und unser Scheitern berichten.

\section{Kassel hat ein ,Platzproblem.}

Wie in vielen anderen Städten fehlt es an Wohnraum für Menschen mit niedrigem Einkommen. Freiräume für Projekte alternativer Kultur und politischen Engagements verschwinden. Die Versuche, Teilhabe und Partizipation im öffentlichen Diskurs um Stadtentwicklung zu verankern, stehen einer Politik gegenüber, die den wirtschaftlichen Aufschwung und den touristischen Ausbau proklamiert und unter dem Deckmantel des kommunalen Schutzschirms sukzessiven Sozialabbau betreibt. Auf der einen Seite steht der Ausbau der Museumslandschaft (z. B. Brüder Grimm-Museum und UNESCO-Weltkulturerbe „Bergpark Wilhelmshöhe“). Auf der anderen Seite die Verdrängung alternativer Kulturprojekte aus dem Südflügel des Hauptbahnhofes zugunsten des neuen Fraunhofer Instituts und der Verlust 
der Kulturfabrik Salzmann. Auf der einen Seite der Bau von Stadtvillen und der enorme Verlust sozialgebundener Wohnungen. Auf der anderen Seite der mahnende Ruf von Sozialverbänden nach günstigem Wohnraum und aktuell die Forderung nach dem Recht auf Wohnen in der Stadt für alle.

Innerhalb dieses Szenarios werfen wir einen Blick auf die Universität Kassel mit ihrem aktuellen Bauvorhaben (Campuserweiterung Nord). In der Auseinandersetzung um einen Quartiersplatz auf dem Universitätsgelände prallt die neoliberale Politik und Praxis von Verwaltung und Planung auf eine alternative, solidarische und sehr heterogene Gemeinschaft von Nutzer*innen. Es geht um Wissen, Macht und Aneignung. Das Große spiegelt sich im Kleinen.

\section{Freiraum und Aneignung an der Reformhochschule Kassel}

Die Universität Kassel wurde in den 1970er Jahren als Gesamthochschule Kassel (GhK) gegründet und verstand sich als Reformhochschule. Erst 1993 wurde die ,Universität' in den Titel eingefügt und 2003 die ,Gesamthochschule' gestrichen. Ihre ersten Jahrzehnte waren geprägt von vielen Disputen. Man wollte das Bildungssystem revolutionieren und überhaupt

\section{Lageplan Uni Kassel in der Stadt}

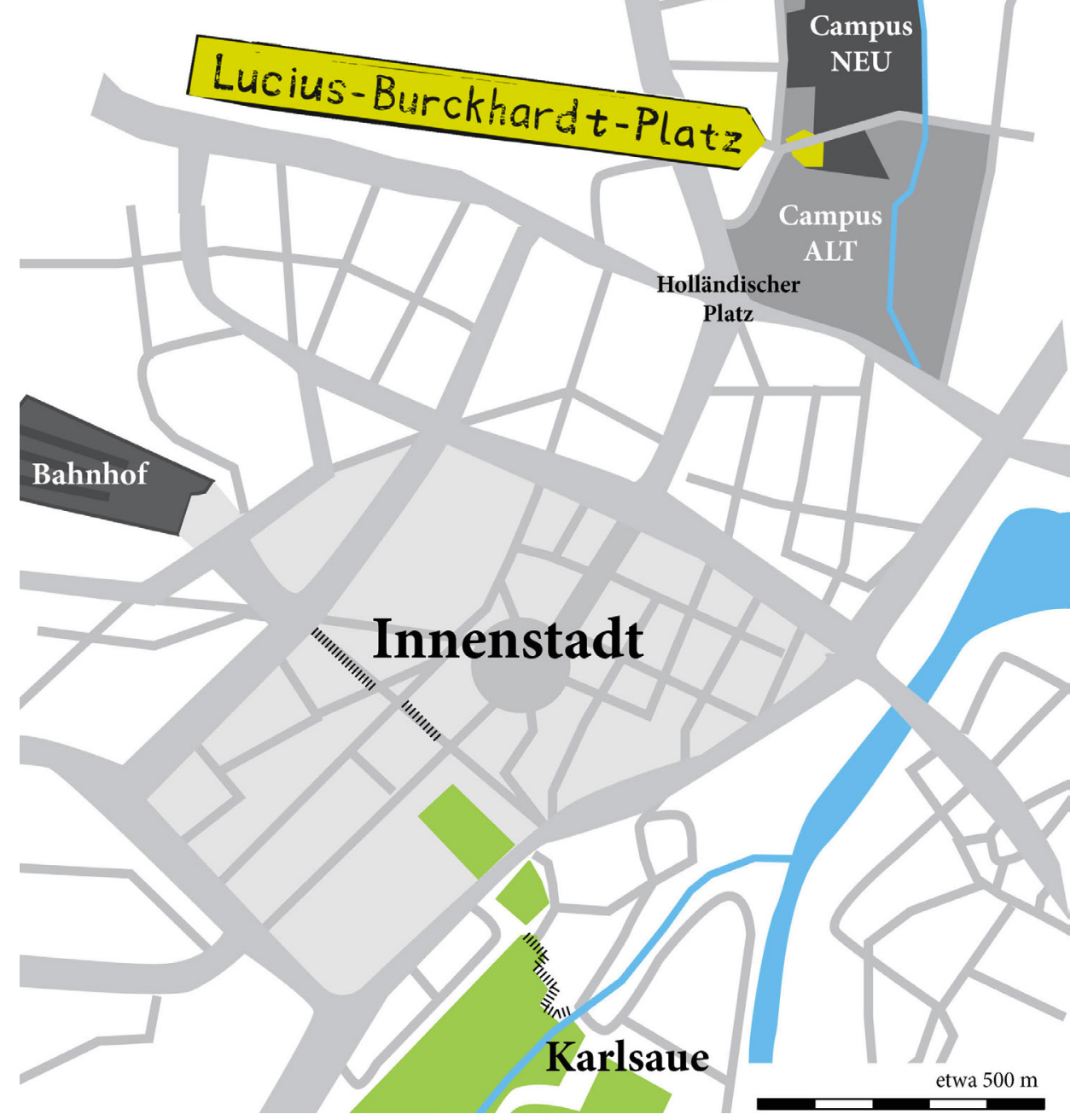

Abb. 1 Lageplan der Universität in der Stadt (Quelle: Archiv Initiative LuciusBurckhardt-Platz bleibt! Kassel) 
Abb. 2 Fabrik

Henschel und Sohn

Cassel 1911 (Quelle:

Henschel \& Sohn

1920: Denkschrift

aus Anlass des

Hundertjährigen

Bestehens der

Maschinen- und

Lokomotivfabrik

Henschel \&

Sohn Cassel und

der Vollendung

der Lokomotive

Fabriknummer 10000,

Cassel 1910.)
Abb. 3 Aktion

Daxner Linde 1988

(Abb3a, links) und

Aktionsbäume 1977

(Abb3b, rechts)

(Quelle: ASL-Grauer

Raum, bearbeitet

von Traudel, Kassel,

Mai 2011)

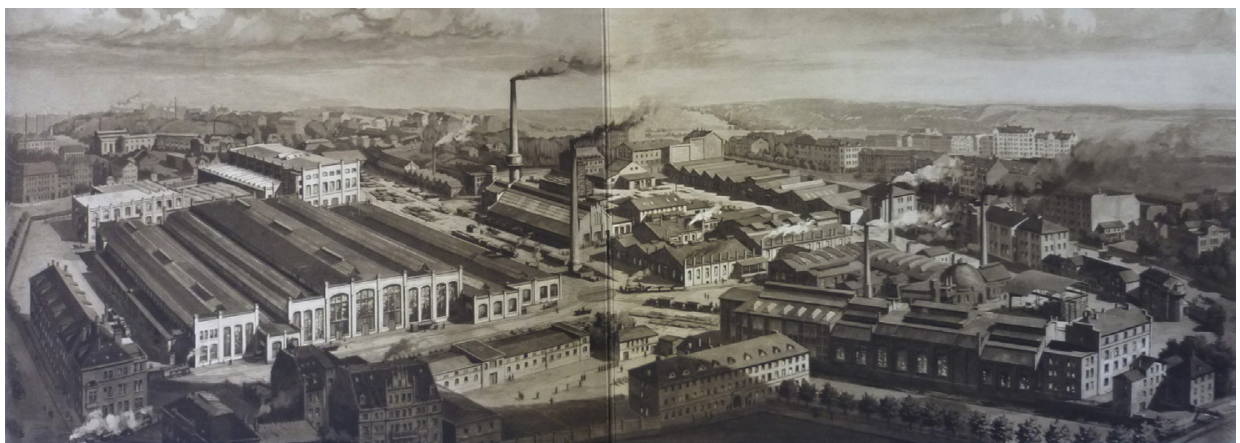

alles anders machen. So siedelte man die Gesamthochschule Ende der 1970er Jahre nicht wie damals üblich am Rande der Stadt, sondern auf einer innerstädtischen Konversionsfläche, dem ehemaligen Gelände der Firma Henschel \& Sohn am Holländischen Platz, an. Wo früher Lokomotiven sowie Rüstungsgüter und später auch Omnibusse produziert wurden, entstand nun ein neuer Campus nordöstlich der Innenstadt. (vgl. Abb. 1) Schon bei der Ansiedlung am Holländischen Platz gab es Auseinandersetzungen zur Campusgestaltung. Auf dem ehemaligen Industriegelände standen große Werkhallen, die einige Gründer*innen und Studierende als erhaltenswert ansahen und für die Ideen zur Umnutzung im Raum standen. (vgl. Abb. 2) Durch den gekonnten nächtlichen Einsatz einer Abrissbirne wurden Fakten geschaffen (akute Einsturzgefahr und Abriss in den Morgenstunden) und weitere Auseinandersetzungen über den Erhalt im Keim erstickt. Der neuen Bebauung stand somit nichts mehr im Weg.

Während die bildungspolitischen Utopien bald in der Realität des deutschen Bildungswesens begraben wurden, setzte sich die aktivistische Tradition der Studierenden, die zusammen mit Mitarbeiter*innen und Nachbar*innen Freiräume errichteten und das Vorgehen von Ministerium oder Präsidium scharf kritisierten, weiter fort. 1977 wurden Aktionsbäume gepflanzt, verbunden mit der Forderung nach Beteiligung bei der Außenraumgestaltung des neuen Campus der damaligen GhK. (vgl. Abb. 3b) In den 1980er Jahren wurde ein reformfreudiger Präsidentschaftskandidat (Herr Daxner) vom Ministerium abgelehnt. Daraufhin streikten Angestellte und Studierende, und pflanzten zusammen mit Joseph Beuys die, Daxner-Linde.. (vgl. Abb. 3a) Ein geplanter Park (eigentlich eine Ausgleichsfläche für die dichte neue Bebauung auf dem Campus) wurde trotz starker Proteste durch Parkplätze (,unter Baumdach') ersetzt. Das Projekt für experimentelles Wohnen auf dem Campus (Bauwagenplatz) wurde von Studierenden initiiert und belebt, bis es auf Anweisung des Präsidiums in den 1990er Jahren gewaltsam
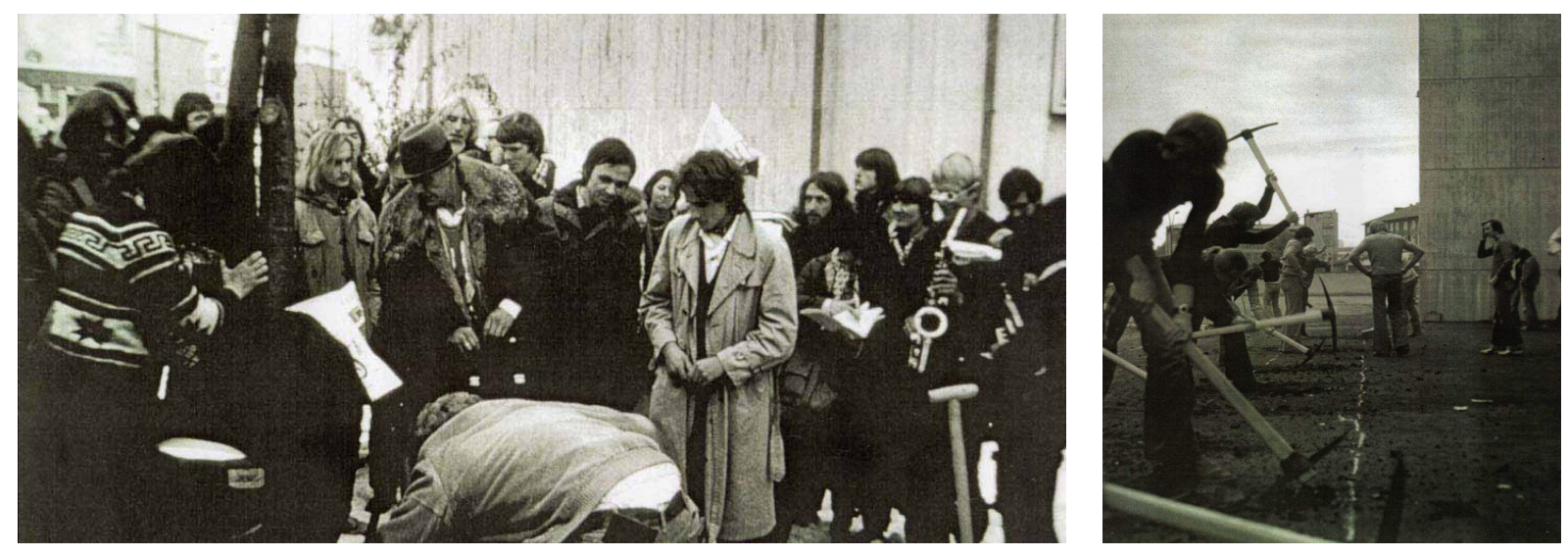
abgeräumt wurde. Die Gestaltung des Campus wurde lange von Studierenden und Mitarbeiter*innen selbst in die Hand genommen oder kritisch durch Aktionen kommentiert. Das hat ebenso Tradition in Kassel wie das ablehnende Gebaren seitens der ,herrschenden“ Planenden auf institutioneller Ebene.

\section{Die Universität und der Stadtteil...}

Die junge Hochschule formulierte über Jahrzehnte das Anliegen, dass „auch an das stadtteilöffentliche Interesse gedacht werden" sollte und proklamierte ,in baulich- und räumlicher Hinsicht immer den Anspruch einer Quartiersoffenheit“ (in der Kasseler Hochschulzeitung Publik von 1992/7: 8). Diese Offenheit wurde vor allem im Nordteil des Campus an der Schnittstelle zum Quartier Nord-Holland (Nordstadt) gewünscht. (vgl. Abb. 4) Nach dem Weggang der Firma Henschel und Sohn und anderen Betrieben galt es diesen von Arbeiter*innen und Migrant*innen geprägten Stadtteil ,wiederzubeleben'. Die Gesamthochschule wurde dafür als wichtiger Hoffnungsträger angesehen, wirkte aber zum Beispiel in Bezug auf die Mieter*innenstruktur im Stadtteil letztlich nur in einem sehr begrenzten Radius (wenige Nebenstraßen). Die Studierenden zog es damals vor allem in den Stadtteil „Vorderer Westen“, der dadurch in den 8oer und 9oer Jahren deutlich sichtbar gentrifiziert wurde.

Das städtebauliche Konzept für den Campus (1980er Jahre) sah nicht nur einen zentralen Eingang am Holländischen Platz mit diagonaler Wegeführung bis zur Bibliothek und zur Mensa vor. Der Campus hat auch heute noch die Anmutung einer postmodernen Kleinstadt, in der Seminarräume, Fachbereiche, studentisches Wohnen, der AStA und die freien Referate bunt

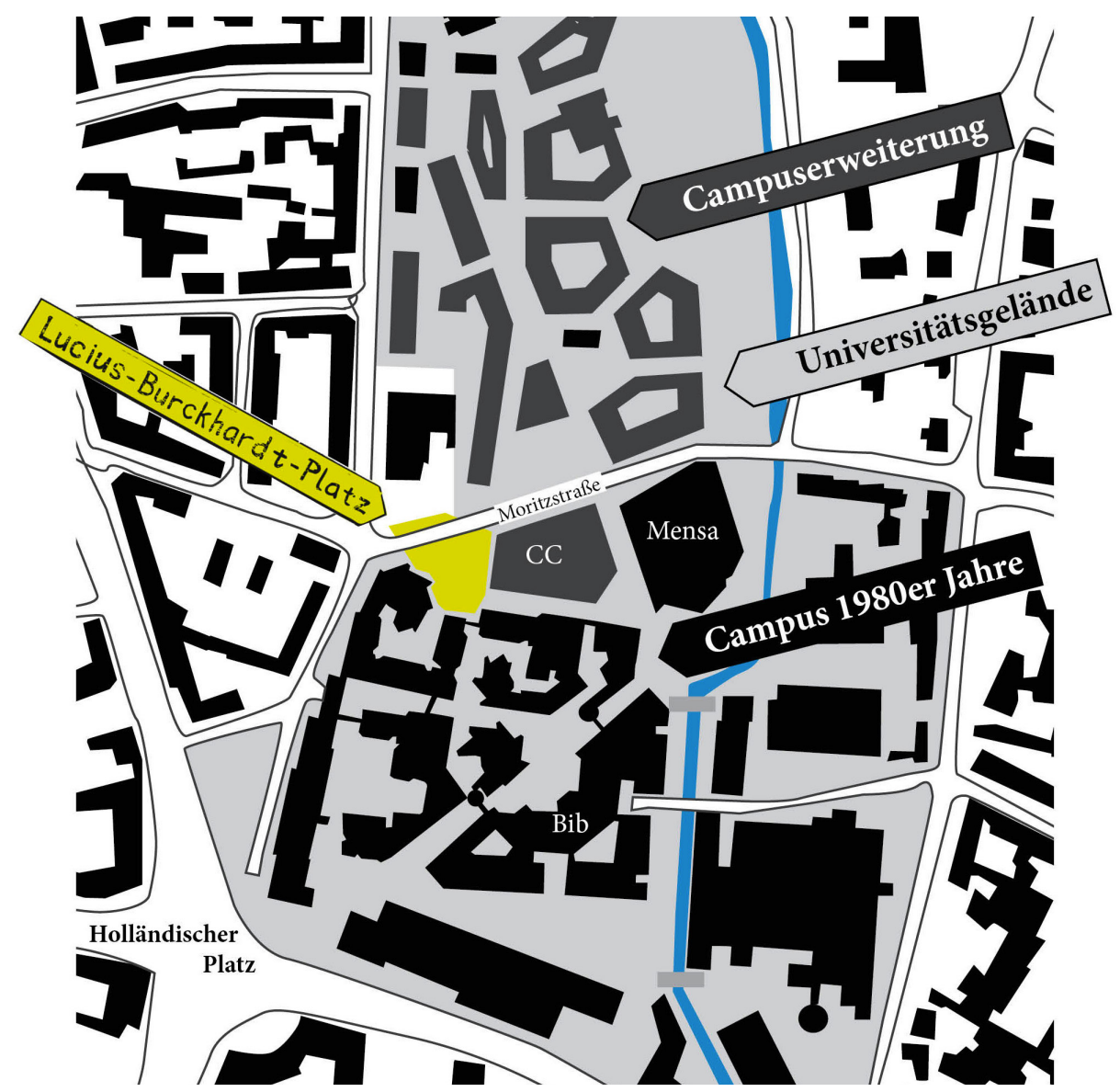

Abb. 4 Die Universität und der Stadtteil (Quelle: Archiv Initiative LuciusBurckhardt-Platz bleibt! Kassel) 


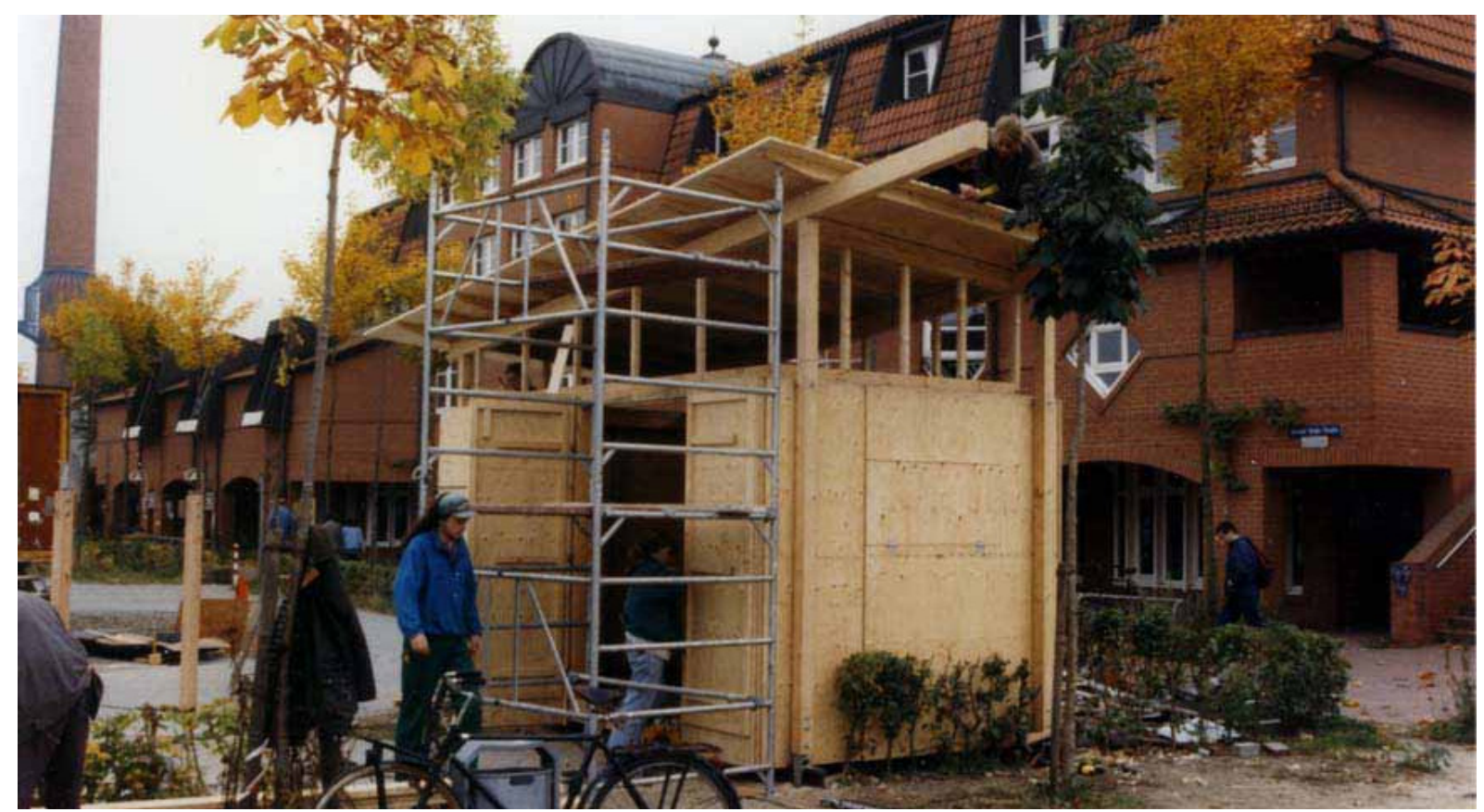

Abb. 5 Bau der Fahrradwerkstatt 1996 (Quelle: Archiv Fahrradwerkstatt des AStA Kassel) verstreut sind. Neben zentralen Fußwegen mit Laubengängen gibt es unzählige andere Durchgänge, Brücken und Treppen, über die man auch kleine Innenhöfe und Dachterrassen erreichen kann. Die Randbebauung ist zum Teil kleinteilig und immer wieder mit Öffnungen versehen, die mal als Tor, mal als Tunnel durch ein Gebäude gestaltet sind. Diese Öffnungen sind die Scharniere, an denen sich der alte Campus mit dem Stadtteil verbindet und eine gute Durchlässigkeit ermöglicht.

\section{...treffen sich auf einem Platz}

Eine der zentralen Schnittstellen zwischen Campus und Stadtteil ist ein kleiner Platz unweit der Mensa, auf zwei Seiten eingefasst von durchlässigen Gebäuderiegeln mit Studi-Wohnungen, Büro- und Seminarräumen und einem kleinen Café. Daneben ein riesiger Parkplatz (heute der Standort des neuen Campus Centers) und die Moritzstraße. Auf dem Platz stehen kleine Linden und vor dem Café hat der Betreiber einen Biergarten mit Hainbuchenhecken umpflanzt. Ein Kiosk findet sich um die Ecke an der Kreuzung.

Im Laufe der 1990er Jahre wird das Café nach einem Streik übernommen und unter Selbstverwaltung als ,Café desasta' weitergeführt. Eine ebenfalls selbstverwaltete Fahrradwerkstatt besetzt als Statement gegen die autogerechte Planung in Kassel zwei Parkplätze am Rand des Platzes. Als kleiner ökologischer Versuchsbau in Holzskelettbauweise, der jederzeit umgesetzt werden kann (fliegender Bau), wird die Werkstatt von Studierenden des Fachbereichs ASL (Architektur, Stadtplanung und Landschaftsplanung) selbst gebaut. (vgl. Abb. 5) Die Fahrradwerkstatt ist ein Ort, wo Studierende und Bewohner*innen der Nordstadt das eigene Fahrrad mit Hilfe und Unterstützung reparieren können. Materielle Unterstützung erfolgt dafür von umliegenden Betrieben. Finanzielle Unterstützung erhalten die Studierenden von dem Studierendenparlament (StuPa), dem Fachbereich ASL, der Gesamthochschule Kassel und dem Studentenwerk Kassel.

Auch die Gestaltung des Platzes übernehmen die Studierenden zu dieser Zeit selbst: Sie ersetzen im Zuge eines studentischen Projektes die größtenteils 


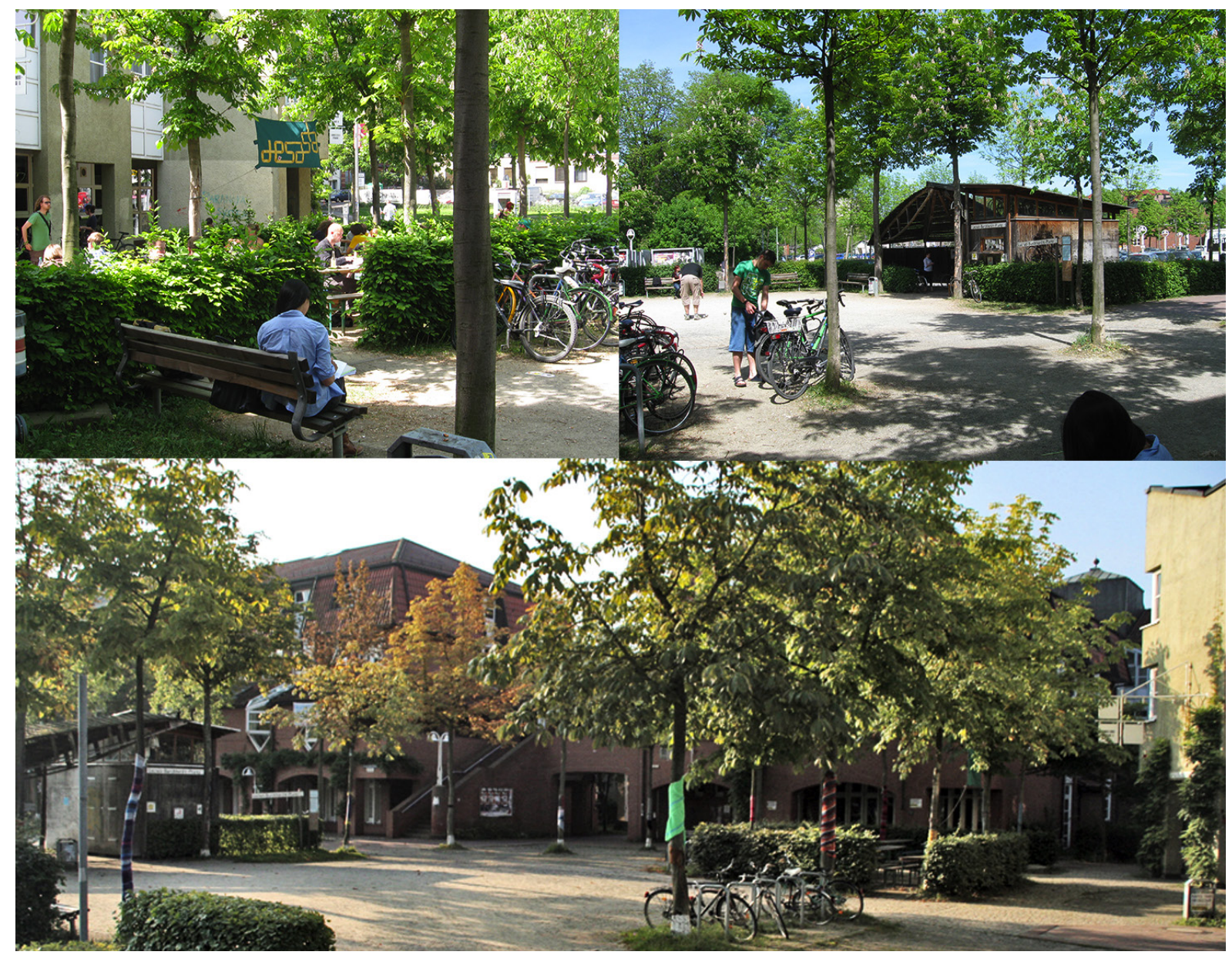

abgestorbenen Linden durch Kastanien und betreiben Aufwuchspflege. Das Café desasta pflegt die Hainbuchenhecken um ihren Biergarten. Es entsteht hier ein kleiner Ruhepol, an dem man Kaffee trinken, Zeitung lesen, Gespräche führen oder kurz innehalten kann. Die großzügige restliche Fläche Abb. 6 Der LuciusBurckhardt-Platz (Quelle: Marina und Alexander) des Platzes ist mit feinem Schotter (wassergebundene Decke) bedeckt und ebenfalls durch Hecken und Bäume eingerahmt. Diese Art von Bodenbelag ermöglicht eine einfache Nutzung, ist aber auch gleichzeitig durch ein geringeres Rückstrahlungsvermögen als andere Versiegelungsmaterialien (wie zum Beispiel Plattensteine, die nun überall auf dem neuen Campus verbaut werden) eine wichtiger Beitrag zum Mikroklima des Platzes (vgl. Blume/ Horn 2011, 162). An den Rändern stehen Bänke und oft begangene Wege sind gepflastert. Hier und da wächst etwas Grünes durch den Schotter, dort, wo er nicht so intensiv begangen wird (Spontanvegetation). (vgl. Abb. 6)

Der Platz wird zu einem beliebten Treffpunkt von Menschen aus der Universität, dem Quartier und der Stadt. Hier wird zusammen an Fahrrädern geschraubt, es werden Feste gefeiert und Boule gespielt. Die Räume des Cafés können auf Anfrage auch außerhalb der Öffnungszeiten genutzt werden. Der Platz am Rande des Campus bildet an dieser Stelle keine Mauer, keinen Zaun und vor allem keine Grenze, zementiert also keine Separierung von Universität und Stadtteil. Es ist ein Quartiersplatz für alle. Baumbestanden, behutsam zoniert, überschaubar, lesbar, besitz- als auch besetzbar durch mobile Bänke und Stühle. Ein Ort, an dem man sich willkommen fühlt, an dem man etwas machen oder anderen zuschauen kann. Die Hecken erhalten dort Öffnungen, wo sie von Menschen ,erlaufen' werden, die Stühle des Cafés wandern der Sonne nach über den Platz. Ein Freiraum, der zugleich 
als Durchgangsort fungiert - allerdings einer, bei dem das Tempo abnimmt. Kaffee, Politik, Sonnenbaden und Schattensitzen, Bier oder Börek, Boule und Bla - ein offener Möglichkeitsraum bestimmt das Programm bis 2011.

\section{Die Campuserweiterung:}

\section{Von neuen Bildungslandschaften und wie sie geplant werden}

Mit dem 2007 in Wiesbaden beschlossenen „Hochschul-Entwicklungsund Umbauprogramm: RundErneuerung, Konzentration und Ausbau von Forschung und Lehre“ (HEUREKA) regnet es in Hessen Fördermittel in Höhe von vier Milliarden Euro für den Ausbau und die Sanierung von insgesamt dreizehn Hochschulen. Damit kann nun auch der Campus der ehemaligen Reformhochschule in Kassel erweitert werden. Mit einer rasant steigenden Anzahl von Studierenden (1991: 16.000; 2015/16: 24.385) ist der alte Campus längst zu klein geworden. Gleichzeitig hat die Universität seit ihrer Entstehung einen fundamentalen Wandel vollzogen, der sich nicht nur in ihrer Umbenennung zur Universität im Jahre 2003 manifestiert, sondern auch in ihren Ausbauplänen widerspiegelt. Ziel des 2005 beschlossenen Baustrukturkonzeptes, das nun umgesetzt werden soll, ist auch die Zentralisierung von Einrichtungen und Fachbereichen auf dem Campus am Holländischen Platz, die vormals dezentral in Kassel verstreut waren. Konträr zum alten Campus sieht das neue städtebauliche Programm dabei jedoch klare Gebäudeformen und getrennte Nutzungszuweisungen vor. Das neue Wohnheim des Studentenwerks und die Kita, das Selbstlernzentrum, der Science Park (,für innovative Start-up-Unternehmen aus allen Bereichen der Universität"), das Campus Center und einige Fachbereiche sollen nun als große Solitäre realisiert werden.

Die Ausschreibung und Entwicklung der Konzepte für die Hochschulerweiterung erfolgt 2008 ohne nennenswerte Einbindung der Anwohner*innen. Die Entwürfe werden zwar kurz ausgestellt, die Begründung für die Auswahl der Sieger*innen des zweistufigen, beschränkten Ideen- und Realisierungswettbewerbes (Architektur: raumzeit; Landschaftsplanung: K1 Landschaftsarchitekten) aber nicht im Stadtteil öffentlich präsentiert, erläutert und diskutiert. Allein der Fachbereich Architektur, Stadtplanung und Landschaftsplanung widmet sich in einer Abendveranstaltung dem Gewinnerentwurf. Der Fokus liegt dabei auf dem neuen Gebäude für den Fachbereich, welches (wie auch das Campus Center und die gesamte Freiflächenplanung) ebenfalls bei dem Wettbewerb ausgeschrieben war. Auf dieser Entwurfsebene endet dann auch die Möglichkeit der Einsichtnahme in detailliertere Planungen. Die spätere Weiterentwicklung des Siegerentwurfs und die Ausschreibungen für die restlichen einzelnen Gebäude wurden ohne Einbeziehung der Öffentlichkeit abgeschlossen und umgesetzt. Nach mehrmaligen gescheiterten Versuchen des Ortsbeirates Nord-Holland, noch Änderungswünsche im neuen Bebauungsplan einzubringen, wird dieser dann von ihm abgelehnt, was jedoch keine Auswirkung auf den positiven Beschluss der Stadtverordnetenversammlung hat.

Das große Planungsvorhaben „Campuserweiterung Nord“ besteht zu diesem Zeitpunkt nur auf dem Papier, seine Bedeutung für die Quartiersrealität vor Ort sickert nur langsam durch. Die Tragweite der Planungen ist für viele 
Menschen schwer vorstellbar. Wo im Zuge des Hochschulausbaus in den 1980er und 9oer Jahren die Bauvorhaben noch transparent vorgestellt und in Dauerausstellungen präsentiert wurden, findet heute kaum noch eine direkte Kommunikation statt. Um dem Anspruch von Transparenz zu genügen, bedient man sich einiger weniger pdf-Plan-Dateien und minimalistischer Renderings auf der Webpräsenz der Universität. Die Zustimmung in den internen Gremien erfolgt anscheinend ebenfalls nur auf Grundlage kleiner Präsentationen und ohne über Details zu diskutieren. Diese Art der Vermittlung ist freilich Teil einer ausschließlichen Top-DownKommunikation, einer oberflächlichen und plakativen Informationspolitik, die Entscheidungsträger*innen und Nutzer*innen notwendiges Wissen vorenthält und keinen Diskurs vorsieht. Weder bei der Architektur der neuen Gebäude, die kaum mehr Bezug zu den Bestandsgebäuden der Hochschule und des Stadtteils haben, noch über den Umgang mit Freiräumen, Menschen und die Art und Weise, wie die Planungsprozesse gestaltet sind.

Der Quartiersplatz mit der Fahrradwerkstatt und dem Café desasta liegt nun nicht mehr am Rande des alten Universitätsgeländes, sondern rückt in dessen neue prominente Mitte, in die direkte Nachbarschaft mit dem neuen Herzstück der Universität, dem Campus Center. Dieses steht als expressiver Bau in Alu und Beton Pate für die neue Bildungslandschaft und beherbergt nicht nur Hörsäle und Seminarräume, sondern auch die zentralen Beratungsund Serviceeinrichtungen der Universität und des Studentenwerks sowie eine Sparkassenfiliale. Es ist auch der neue repräsentative Veranstaltungsort für Kongresse und andere Großveranstaltungen.

\section{Alternativlosigkeit und Definitionsmacht}

Als die ersten Bilder und Pläne vom Neubau des Campus Centers veröffentlicht werden, wird uns Nutzer*innen des Quartiersplatzes klar, dass die geplanten Baumaßnamen auch weitreichende Konsequenzen für den Platz haben werden. Da weder die Mitarbeiter*innen der Fahrradwerkstatt, noch die des Café desasta bisher über Umbaupläne informiert wurden, ging man bis dato davon aus, dass der Platz nicht Teil der Neuplanungen sei. Trotzdem beginnen wir zu recherchieren und beschaffen uns detaillierte Pläne. Daraus geht hervor: Die Fahrradwerkstatt soll dem Campus Center weichen. Außerdem ist der gesamte Platz während der dreijährigen Bauzeit als eingezäunte Baulogistikfläche eingeplant. Obwohl er mit seinem gewachsenen Gefüge inzwischen seit Jahrzehnten elementarer Bestandteil der Quartiers- und Hochschulkultur ist, soll er während der gesamten Bauphase nicht nutzbar sein und danach als neuer Vorplatz des Campus Centers gestaltet werden. (vgl. Abb. 7)

Wie begegnet man einer solchen bürokratischen und autoritären Planung? Mit den ersten Nachfragen bei der Bauabteilung wird deutlich: Wir stehen scheinbar vor alternativlosen Tatsachen. Die Baulogistikfläche sei unverrückbar, weil sowieso schon zu knapp bemessen. Die Fahrradwerkstatt könne ja irgendwo anders auf dem alten oder neuen Campus stehen. Und das Café hätte dann in drei oder vier Jahren einen schönen neuen Platz vor der Tür, der ja auch repräsentativ sein solle. Damals noch ungesagt, aber deutlich spürbar: Die Bedeutung und die gewachsene Qualität des Platzes werden nicht nur negiert, weil es kosteneffizienter oder einfacher ist, sondern 


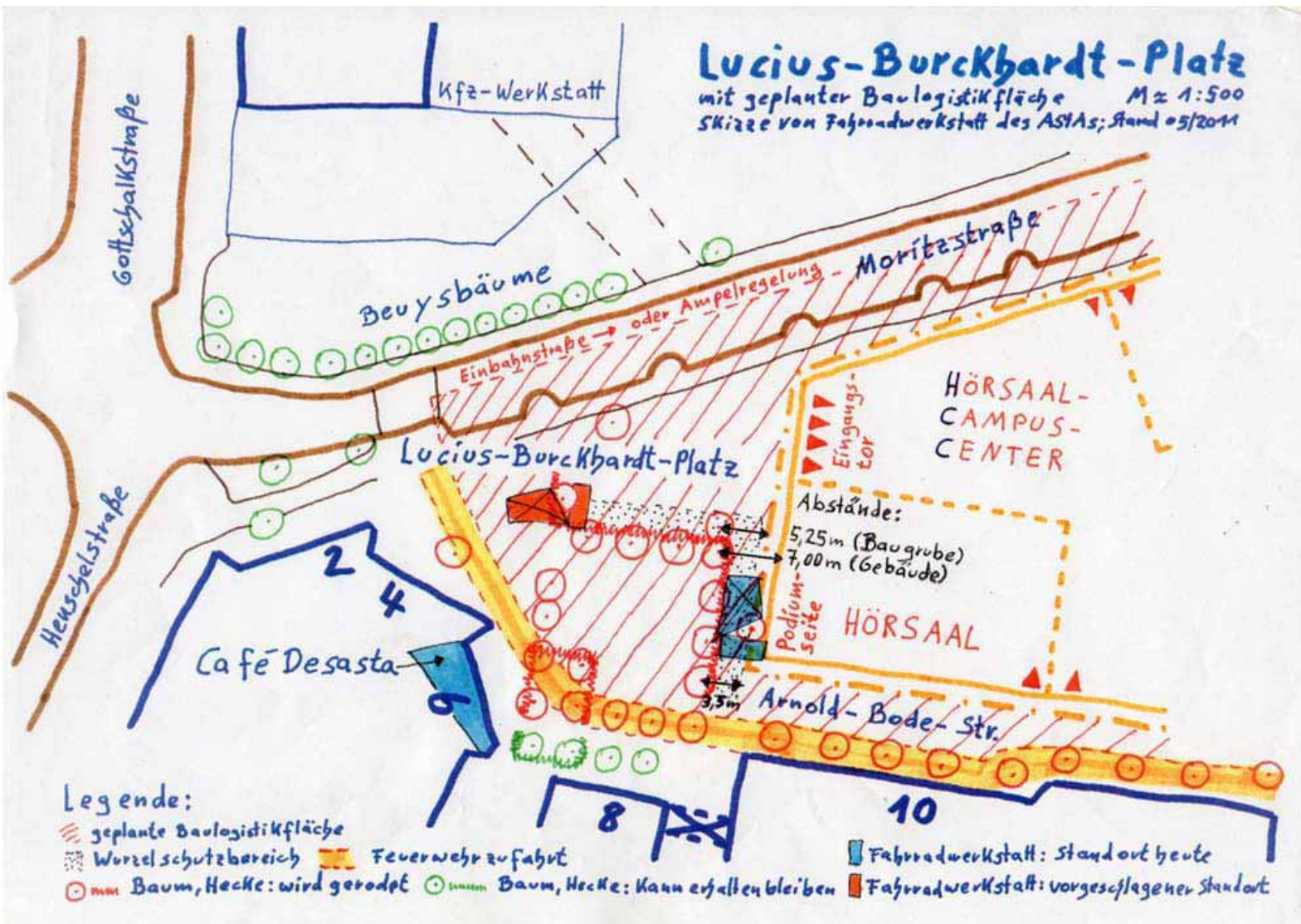

Abb. 7 Der Platz und die Baulogistikfläche (Quelle: Archiv Fahrradwerkstatt des AStA Kassel. Erstellt von Walter) sie sind auch nicht mehr erwünscht. Die Platzgestaltung aus den 1980 und 1990er Jahren entspricht einem Planungsverständnis, das weit über die einfache Setzung verschiedener baulicher Elemente (Bäume, Bänke, Hecken, Formbarkeit) hinausgeht. So hat der Platz (durch die Bäume) am Rand und über dem Biergarten ein Dach. Er hat Wände (aus Hecken und Bänken), die ihn einrahmen. Das Design orientiert sich vor allem an der Nutzung des Platzes: Das heißt: Nur dort, wo viel Verkehr ist, werden Wege gepflastert, der Rest bleibt frei. Hecken sind dort durchlässig, wo es durch Nutzer*innen eingefordert wird. Diese eher auf den Prozess der Nutzung reagierende Planung wird nun als unzeitgemäß dargestellt und soll dem neuen gestalterischen Leitbild der Universität angepasst werden. Dazu gehört auch die Art der Aneignung und Bespielung des Platzes. Wo voraussichtlich in Zukunft Massen von Studierenden auf der Jagd nach dem nächsten Credit Point passieren sollen, scheint ein Ort, der individuelle Aneignung und Verweilen ermöglicht, im wahrsten Sinne des Wortes fehl am Platze. Fortan wird der Platz von der Bauabteilung der Universität nur noch als „Baulogistikfläche am Campus Center“ oder „westlicher Vorplatz des Campus Centers“ bezeichnet.

$\mathrm{Zu}$ diesem Zeitpunkt (2011) beginnen wir als eine Initiative von Platznutzer*innen damit eine Gegenmacht aufzubauen. Als Bewohner*innen der umliegenden Quartiere und als Studierende und Beschäftigte an der Universität Kassel wollen wir gemeinsam für den Erhalt des Platzes kämpfen und die kolportierte Alternativlosigkeit nicht hinnehmen. Wir entscheiden uns dafür, der Definitionsmacht der Universität eine eigene gegenüberzustellen. Was einen eigenen Namen hat, kann man besser verteidigen. Um auf die besonderen Qualitäten und andere Formen der Planung hinzuweisen, beziehen wir uns auf die Ideen eines bekannten ehemaligen Kasseler Professors. „Lucius-Burckhardt-Platz“ ist der (zumindest im Alltag schnell etablierte) neue 


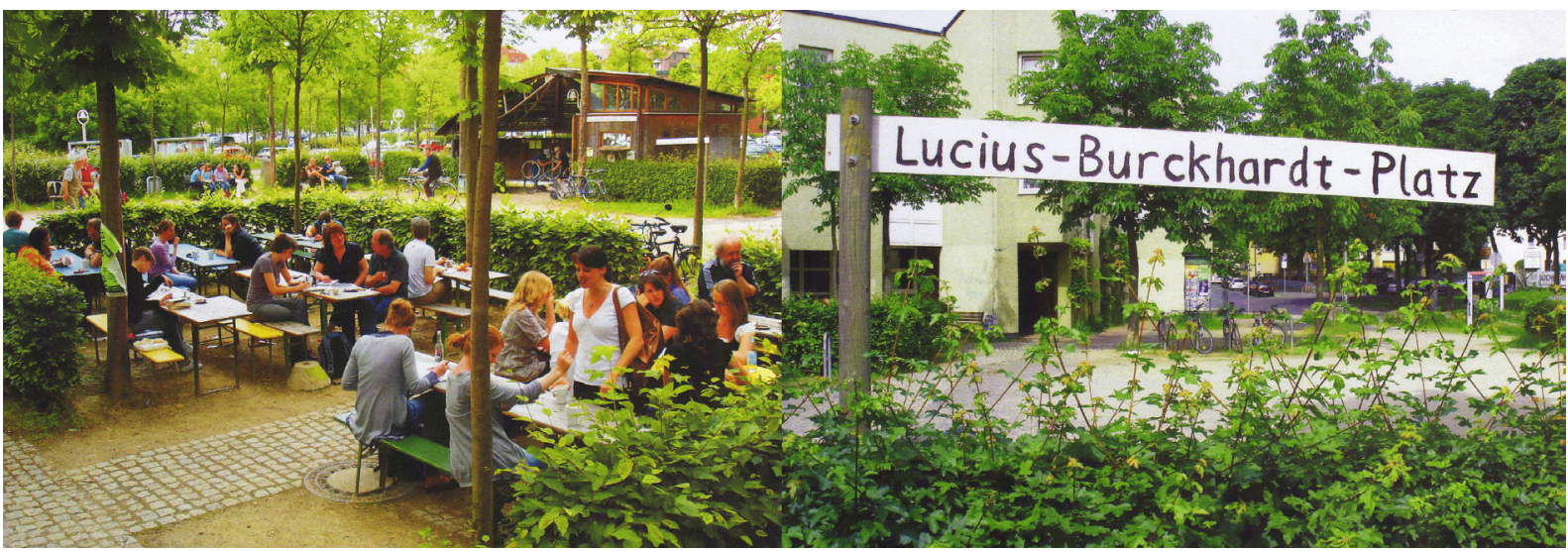

Name des Platzes, der vorher keinen hatte. (vgl. Abb. 8) Unser Namenspate ist berühmt: Lucius Burckhardt gehörte nicht nur zur Gründungsgeneration des integrierten Studienganges Stadt-/Landschaftsplanung und hatte eine Professur für Sozialökonomie urbaner Systeme in Kassel inne, sondern verfolgte auch eine partizipatorische und kommunikative Einbindung der Menschen in Planungsprozesse. Bekannt sind auch seine behutsamen Lösungsansätze für den Umgang mit Problemen in bestehenden räumlichen und sozialen Gefügen: „Jeder Übelstand sollte mit derjenigen Strategie bekämpft werden, die den dafür kleinstmöglichen Eingriff darstellt und damit am wenigsten unerwartete und schädliche Folgen hat." (Burckhardt in Ritter/Schmitz 2013: 148) Der „kleinstmögliche Eingriff“ ist ein Gedanke, welcher sich beispielhaft dafür verwenden lässt, die zweckrationale und negierende Planungshaltung der Bauabteilung an der Universität Kassel sichtbar zu machen und ihr etwas entgegensetzen, indem man das Problem klar benennt.

Lucius Burckhardt deutete den „kleinstmöglichen Eingriff“ als Regulativ und meinte damit das Vorgehen (Strategie) bei einem planerischen Eingriff (ebd.: 117). Gleichsam war es auch eine Kritik an den gängigen „Mechanismen der Beschlussfassung“, die oftmals eine Legitimation durch den Hinweis auf eine Gesamtkonzeption (in unserem Fall die Campuserweiterung) darstellten und als „Teil der Ideologie von Mittel und Zweck“ fungierten (vgl. ebd.: 148f.). Konstruktiv ergänzen kann man diese Kritik durch seine Betrachtungen zum ,unsichtbaren Design“ welches eine unbemerkte Sozialfunktion erfüllt, die sich beispielsweise dadurch auszeichnet, dass sie ein Gesamtsystem und zwischenmenschliche Beziehungen bewusst berücksichtigt.

\section{Eine Frage des Territoriums}

Unsere Initiative erhält ihren Namen: „Lucius-Burckhardt-Platz bleibt!“ und gewinnt immer mehr Unterstützer*innen. Mit dem Namen als Programm kommunizieren wir öffentlich unsere Kritik an der Planung, treten als Gesprächspartner*innen (auch gegenüber der Bauabteilung) auf und informieren in verschiedenen Veranstaltungsformaten über die bevorstehende Zerstörung des beliebten Platzes.

Wir zeichnen Pläne (die Baustelleneinrichtungspläne der Bauabteilung sind für Laien nicht zu entziffern) und hängen sie öffentlich im Café aus. Wir bauen die Umrisse der Baulogistikfläche mit Bauzäunen nach, um deutlich zu machen, wie es in den nächsten drei Jahren aussehen wird. Eine Künstlerin strickt in einer Mitmachaktion die neunzehn Bäume auf dem Platz ein. Im
Abb. 8 Benennung in Lucius-BurckhardtPlatz (Quelle: Archiv Fahrradwerkstatt des AStA Kassel) 
Herbst 2011 werden kurz vor Baustart gemeinsam Lucius-Kastanien eingetopft und verschenkt.

In einem breiten Bündnis mit über 100 Gruppen und Einzelpersonen (der Vollversammlung der Studierendenschaft, dem AStA, den freien Referaten, der Fachschaftskonferenz, dem Ortsbeirat, dem ADFC, Geschäftsinhaber*innen in der Umgebung und vielen mehr) sowie einer Unterschriftenaktion mit über 1505 Unterschriften fordern wir von der Stadt und dem Präsidium den Erhalt des Platzes während der Bauphase und darüber hinaus die Berücksichtigung von bestimmten Planungsgrundsätzen: die partizipative Einbindung der Nutzer*innen bei zukünftigen Planungen und die Integration bestehender Einrichtungen, Nutzungen und Gestaltungsmerkmale. Durch den gemeinsamen Druck und die Unterstützung des Ortsbeirates Nord-Holland (Nordstadt) erreichen wir im Herbst 2011, dass die Fahrradwerkstatt auf die gegenüberliegende Seite des Platzes (auf städtischen Grund) umsiedeln kann und somit weiterhin am Platz und für alle im Quartier sichtbar und erreichbar bleibt. (vgl. Abb. 9) Schwieriger gestalten sich die Verhandlungen über die Einrichtung der Baulogistikfläche. Durch die detaillierte Analyse der bestehenden Pläne und die penetranten Auseinandersetzungen mit der Bauabteilung der Universität und dem Vertreter des Hessischen Baumanagements können wir zumindest einen kleinen Teil des Platzes erhalten. Der Bauzaun und die eigentlich so notwendige Feuerwehrzufahrt werden ein kleines bisschen verschoben, so dass der Biergarten und eine Baumreihe erhalten bleiben können. Eineinhalb Meter weniger für die Baustelle bedeutete für uns den Erhalt eines wesentlichen Bausteins des Platzes über die Bauzeit hinweg.

Mit dem Verbleib der Fahrradwerkstatt und des Biergartens mitsamt seinem Baumdach am Platzkonnte das besondere Flair des Ortes seit dem Baustart

Abb. 9 Umsetzung der Fahrradwerkstatt 2011 (Quelle: Archiv Fahrradwerkstatt des AStA Kassel)

Abb. 10 Der LuciusBurckhardt- Platz ist abgeräumt 2016 (Quelle: Archiv Initiative Lucius-Burckhardt-Platz bleibt! Kassel) 2011 erhalten werden. Trotz Baulärm, Staub und enormer Verkleinerung ist der Lucius-Burckhardt-Platz mit seiner besonderen Atmosphäre nach wie vor Schnittstelle und Treffpunkt von Universitätsangehörigen und Quartiersbewohner*innen. Die Boulespieler*innen sind auf die neue kleine Freifläche vor der Fahrradwerkstatt ausgewichen, die Fahrradschrauber*innen holen ihren Kaffee nach wie vor im Café desasta. Der Bauzaun wird als Schwarzes Brett und Litfaßsäule umgenutzt.

\section{Verleugnung als Strategie}

Trotzdem stehen wir im Sommer 2016 vor den Trümmern des Lucius Burckhardt-Platzes, dessen mühsam über die Baustellenzeit hinüber geretteten
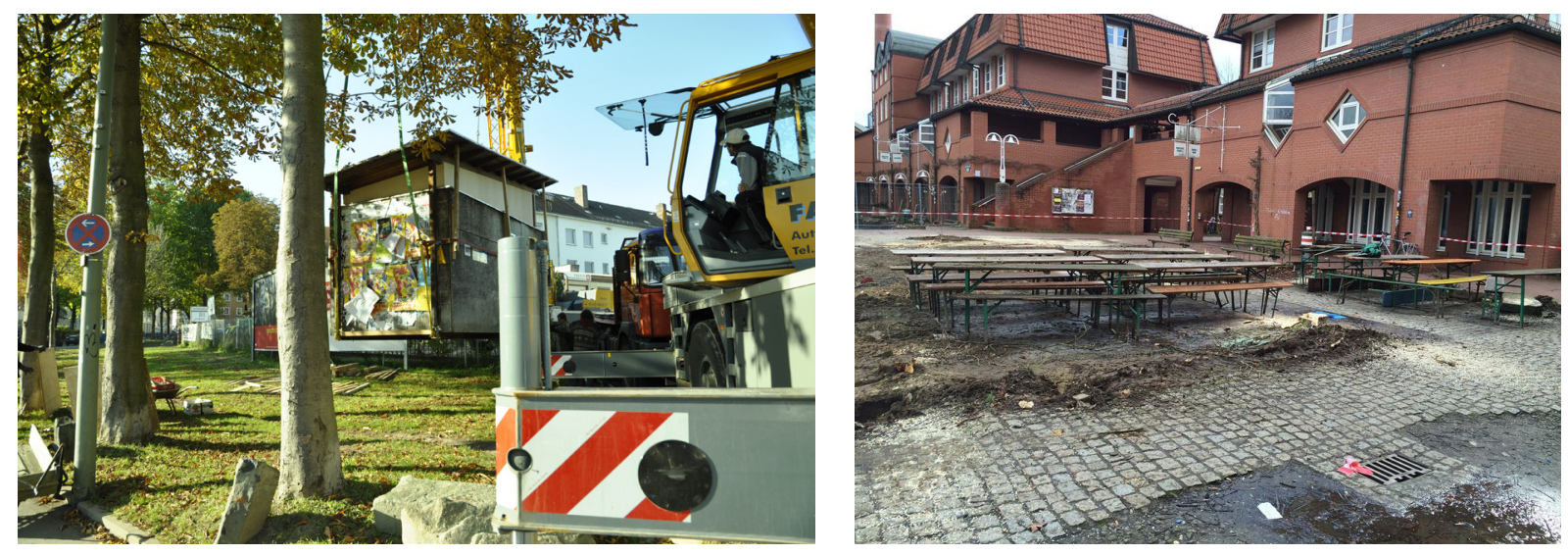
Reste am frühen Morgen im Februar ohne vorherige Ankündigung und entgegen mündlicher Vereinbarungen mit dem AStA zerstört wurden. Die Empörung der Nutzer*innen entlädt sich in einem großen Plenum am Abend des gleichen Tages. Der AStA gibt sich in seinen Pressemeldungen fassungslos, die Mitarbeiter*innen des Café desasta fegen Schotterreste von ihrem Biergartenmobiliar, das nun verloren auf der Brache herumsteht. (vgl. Abb. 10) Noch im Herbst 2015 hatten wir über den Ortsbeirat NordHolland (Nordstadt) bei Stadt und Universität angefragt, wann die Beteiligung bei der Neuplanung beginnen würde, da die Bauarbeiten am Campus Center so gut wie abgeschlossen waren. Während die Stadt darauf verwies, dass sie für den Platz nicht zuständig sei (Landesterritorium), erklärte die Universität, dass erst spät im nächsten Jahr mit der Platzgestaltung begonnen werde.

Nach öffentlichem Protest bekunden das Präsidium und die Bauabteilung nun universitätsintern ihr Bedauern: Es sei irgendwie falsch oder eben nicht an den richtigen Stellen kommuniziert worden. Es sei doch immer klar gewesen, dass der Platz einer kompletten Neugestaltung unterzogen würde. Dass der Wunsch nach dem Erhalt des Biergartens auch seine Einfassung mit Bäumen und Hecken beinhaltete, sei ihnen nicht bewusst gewesen. Es wurde schnell deutlich: Der vermeintliche ,Frieden', der durch den Erhalt während der Bauzeit erkämpft wurde, war eine Illusion. Die Vorstellung, die Arbeit der Initiative vor sechs Jahren hätte dazu geführt, dass die Bauabteilung und das Planungsbüro unsere Forderungen berücksichtigen würden, war eine fundamentale Fehleinschätzung. Sie hatten weder vor, an den Bestand anzuknüpfen, noch die Nutzer*innen bei der weiteren Planung zu beteiligen.

Im weiteren Verlauf der Auseinandersetzung zeigt sich deutlich: Die Initiative hat etwas gemeinsam mit ihrem Platz. Dieser wird in den öffentlichen Verlautbarungen der Bauverantwortlichen der Universität konsequent als „Freifläche westlich des Campus Centers“ bezeichnet (Bauabteilungsleiter Klaus Sausmikat im Campusradio Kassel 2016). Der Begriff der Freifläche suggeriert weder Nutzung noch Funktion oder gar Entstehungsgeschichte. Gleichzeitig wird jegliche Kommunikation von Seiten der Bauverantwortlichen nur noch über den AStA geführt, obwohl dieser auf die Initiative „LuciusBurckhardt-Platz bleibt!“ als offizielle Ansprechpartnerin verweist. Initiative und Platz werden verleugnet.

Einige Nutzer*innen des Platzes beginnen kurz nach der Zerstörung direkt mit Maßnahmen zur Wiederherstellung. Weiden werden anstelle der ausgerissenen Hecke rings um den Biergarten gepflanzt und alle möglichen Gegenstände herangetragen, Sitzmöbel und kleine Rauminstallationen errichtet. Transparente und Flyer werden ausgehängt. Die Auseinandersetzung spitzt sich zu. Nach einem großen Plenum am Platz, bei dem wiederholt der Bedarf nach einer öffentlichen Diskussion geäußert wird, organisiert die Initiative (mit Rückendeckung durch den AStA) eine öffentliche Podiumsdiskussion im Campus Center, auf welcher der neue Präsident (ein Forstwissenschaftler), der Kanzler, der Leiter der Bauabteilung der Universität sowie ein Ortsbeiratsmitglied, ein Student, eine Mitarbeiterin des Café desasta und ein Mitglied der Initiative zu Wort kommen. (vgl. Abb. 11) Der Präsident versucht wortreich, das bisherige Vorgehen als etwas problematisch zu entschuldigen, aber es wird klar: Die Planungen für die Platzgestaltung sind abgeschlossen, das Material bestellt und die Baufirma beauftragt. Spielraum 
Abb. 11 Bild zur

Einladung für die Podiumsdiskussion 2016 (Quelle: Archiv Initiative LuciusBurckhardt-Platz bleibt! Kassel)
Abb. 12 Modelle für die Ideenwerkstatt 2016 (Quelle:

Archiv Initiative LuciusBurckhardtPlatz bleibt! Kassel)

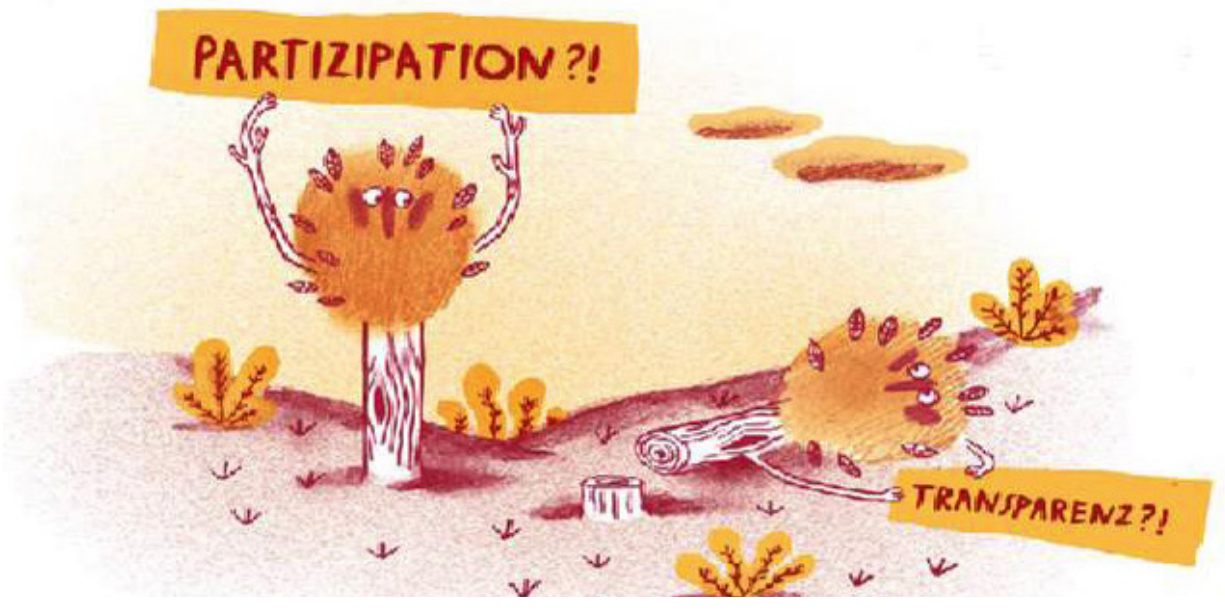

für Beteiligung gibt es trotz einer Entschuldigung für das Vorgehen nicht. Die Universitätsleitung befürchtet schlechte Presse und demonstriert hier Gesprächsbereitschaft, allerdings ohne auf die wiederholten Forderungen von Menschen im Publikum und den Teilnehmer*innen auf dem Podium nach Teilhabe bei der weiteren Bebauung einzugehen. Leider wird dem Kasseler Campus Radio verweigert einen Mitschnitt zu erstellen. Soweit geht dann der Wille nach Transparenz seitens der Leitung doch nicht.

Nach dieser Veranstaltung ist sich die Initiative unschlüssig, wie es weiter gehen soll. Einige Nutzer*innen sprechen über eine Besetzung, andere hoffen auf einen Dialog. Der Bedarf, sich mit der neuen Planung und der aktuellen festgefahrenen Situation zu beschäftigen, bleibt groß. Letztlich entscheidet sich die Initiative dafür eine öffentliche Ideenwerkstatt zu veranstalten. Uns geht es aber vor allem darum, die Qualitäten des ursprünglichen Platzes herauszuarbeiten und dass sich mit dem geplanten Entwurf der Bauabteilung auseinander gesetzt wird. Uns war durchaus bewusst, dass wir damit eigentlich das machen, was wir von der Universitätsleitung einfordern. Neben Mitarbeiter*innen der Universität, Studierenden und Bewohner*innen der Nordstadt nehmen sowohl der Leiter der Bauabteilung als auch ein Vertreter des Planungsbüros an unserer Ideenwerkstatt teil. Im Verlauf der gemeinsamen Diskussion und Begutachtung der von uns angefertigten maßstabsgetreuen Modelle von Bestand und Neuplanung (vgl. Abb. 12) wird deutlich, dass bestehende Strukturen des Platzes ganz bewusst nicht in die Entwürfe mit einbezogen wurden. Obwohl es möglich gewesen wäre, alte Elemente

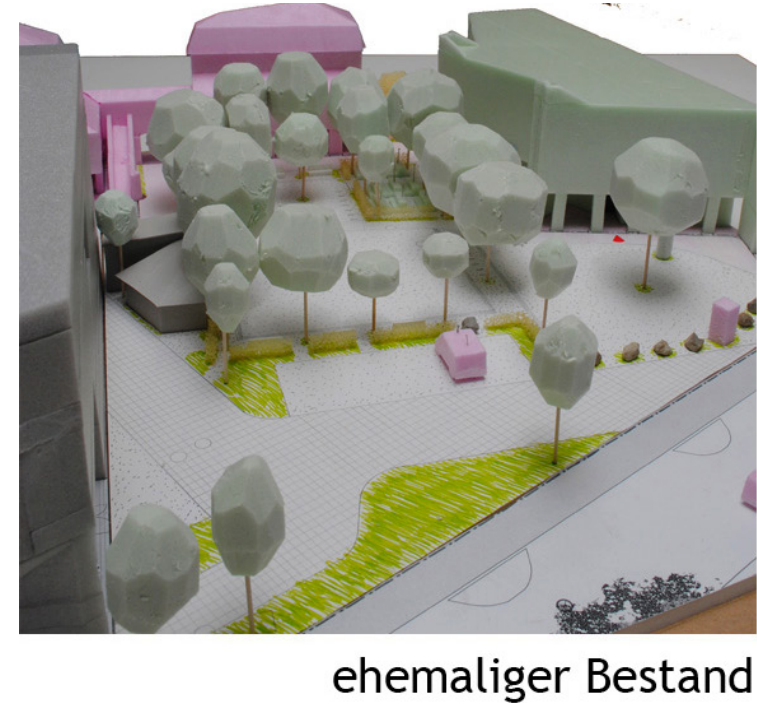

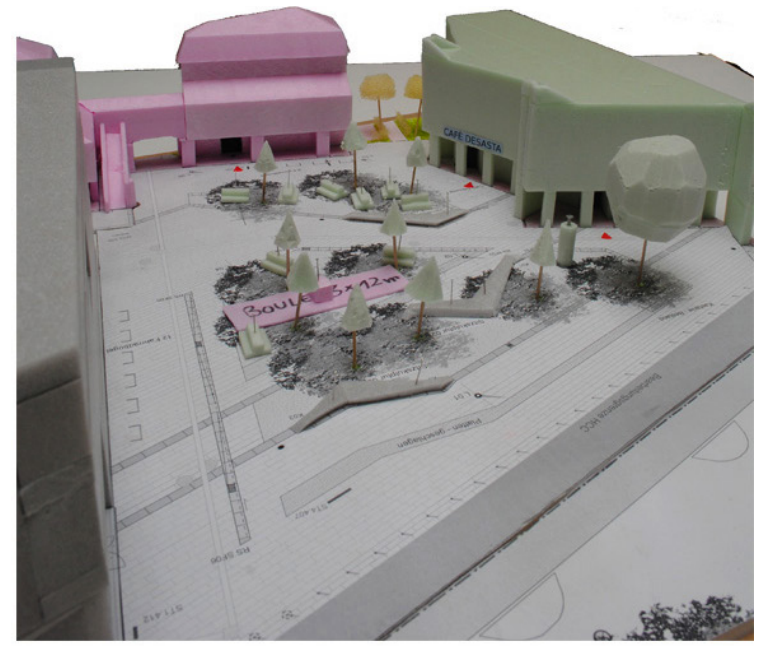

Entwurf 
und Zonierungen wie einzelne Bäume und Hecken zu erhalten und zu integrieren, entschied sich das Planungsbüro dafür, den Platz komplett neu zu gestalten: Einheitlichkeit mit der restlichen Freiflächengestaltung auf dem neuen Campus wird angestrebt. Dieses Vorgehen beruht auf der vom Bauabteilungsleiter im Prozess immer wieder proklamierten These, dass sich die besonderen sozialen Qualitäten des Lucius-Burckhardt-Platzes einfach von allein auf dem neugestalteten Platz wiederherstellen ließen. Dass er damit natürlich nur bestimmte „besondere soziale Qualitäten“ meint, ist uns schon beim Kampf um die Umsiedelung der Fahrradwerkstatt, spätestens aber mit der endgültigen Abräumung des Platzes, klar geworden. Dem alten Platzkonzept (mit einer freien Mitte mit Rückzugs- und Beobachtungsräumen am Rand, die den Platz einfassen) wird ein von Gebäuden gerahmter gepflasterter Platz entgegengesetzt, bei dem die Aufenthaltsorte (wie auf einem Podest) in die Mitte gerückt sind. Nach der Abholzung der vierundzwanzig über zwanzig Jahre alte Bäume werden nun acht neue gepflanzt. Das neue Gestaltungskonzept zeichnet sich vor allem durch seine Endgültigkeit aus. Wird es realisiert, ist der Platz fertig. Dann wird er sich nicht baulich weiterentwickeln können. Anders als bei den Planungen aus den 1980er Jahren wird es hier keine Gestaltung durch Spuren von Nutzer*innen geben. Ergebnis der Ideenwerkstatt ist, dass eventuell noch minimale Änderungen im Bereich des Café desasta vorgenommen werden könnten.

\section{So sieht es heute aus:}

Der Platz ist 2016 endgültig abgeräumt, um keine Anknüpfungspunkte für Proteste zu liefern. Er wird als Freifläche definiert, was ihm jegliche Geschichte und Persönlichkeit abspricht. Die Neuplanungen werden erst auf enormen Druck präsentiert, die Organisation von Veranstaltungen zur Diskussion über diese Pläne übernimmt die Initiative. Sie wird jedoch als außeruniversitäre Gruppe von jeglicher Kommunikation ausgeschlossen. Die Universität baue schließlich keine Stadtplätze und müsse daher nicht die Bürger einbinden, sagte der Leiter der Bauabteilung in der Ideenwerkstatt. Eine späte, aber noch machbare und von vielen gewünschte Beteiligung, sowie eine Neuplanung, würde Kosten verursachen, die für die Universität nicht tragbar seien (Gedächtnisprotokoll 2016). Obwohl die Neubauten Mehrkosten in Millionenhöhe erzeugt haben, sah man sich in diesem Fall nicht in der Lage, auf die Wünsche der dort lebenden und arbeitenden Menschen einzugehen. Mit der Zusage, minimale Änderungen vorzunehmen, vermeiden Hochschule und Planungsbüro eine weitere Schädigung ihres Images.

Bereits 1986 beschreibt Michael Schwarze eine Vorgehensweise, der nicht nur wir Platzfreund*innen zum Opfer fielen:

„Die Strategie der Behörden läuft meist im ersten Schritt darauf hinaus, die Einwände und Argumente der Betroffenen als unsachlich oder fachlich unterbemittelt zu disqualifizieren. Nützt das nichts, so wird die Berechtigung des Protests mit dem Argument bestritten, die Bürgerinitiativen verträten nicht das Allgemein-, sondern ein Sonderinteresse. Auf der dritten Stufe werden dann kleinere Formfehler zugegeben, in bestimmten Details werden Kooperationsangebote 
gemacht. Dieses ,Entgegenkommen“ schafft ihnen ein politisches Alibi.“ (Schwarze in: Andritzky/Spitzer 1986: 416 f.)

\section{Und nun?}

Was wurde erreicht oder besser: Welches Ergebnis kam zustande? Wir haben als Initiative viel voneinander gelernt. Haben Spaziergänge über den Platz und den Campus gemacht, uns Geschichten über seine Entstehung und die Proteste erzählt. Wir haben unser Wissen ausgetauscht. Der Platz ist nicht einfach weg, nur weil dort gerade eine Brache ist. Der Platz ist nicht einfach weg, weil dort jetzt ein durch den,Verwaltungsmechanismus، erzeugtes Abziehbild entsteht. Wir tragen seine Idee und sein Bild in unseren Geschichten weiter. Trotzdem erweist es sich als schwierig für etwas zu kämpfen, das so essentiell für die Benutzung des Platzes war und nun verschwunden ist: seine über Jahre gewachsene und gestaltete bauliche Substanz. Bei den Diskussionen in diesem Jahr zeigte sich aber auch reges Interesse an den restlichen neuen Flächen auf dem neuen Campus. Die Umsetzung der Freiflächenplanung für diesen Teil steht kurz bevor. Vielleicht gibt es hier eine neue Dynamik und neue Stimmen, die ihre Mitbestimmung bei der Gestaltung einfordern?

\section{Was bleibt:}

Die sich immer mehr in den Platz einfräsenden Veränderungen, der Wunsch noch mehr Menschen für die Gestaltung ihrer Freiräume zu mobilisieren und über die Geschichte zu informieren. Wie begegnet man einer Politik der Order von oben und der Tabula rasa? Der Bezug auf den guten alten Burckhardt hat uns ganz konkret und praktisch bisher nicht viel weitergeholfen. Bei der Vermittlung von Inhalten aber schon: Es kam zu aktivierenden Gesprächen mit Menschen auf dem Platz darüber, warum er sich bisher so gut angefühlt hat und was ein behutsames, prozesshaftes Vorgehen für eine Planung bedeutet. Offenbar werden Ideen, von damals', die wir heute auch gerne noch in großen Reden feiern, an einer alternativlosen und sterilen Realpolitik nach dem Motto ,für alle, aber gerade dadurch nicht jeden 'scheitern. Modern muss es heute sein, bitte überschaubar, mit klaren Zuständigkeiten und vor allem im großen Stil, damit hinterher alles zumindest verbal zusammengefasst werden kann... und wenn Proteste laut werden, werden durch die Hintertür Tatsachen geschaffen und in Grimm'scher Tradition Märchen erzählt.

\section{Autor_innen}

Die sieben Textfresser*innen sind ein temporäres Autor*innenkollektiv aus Kassel. Einige setzten sich bereits seit 2011 für den Erhalt des Lucius-Burckhardt-Platzes ein. Alle waren 2016 Teil der Initiative Lucius-Burckhardt-Platz bleibt! initiative_lucius-burckhardt-platz@gmx.de 


\section{Literatur}

Blume, Hans-Peter / Horn, Rainer / Thiele-Bruhn, Sören (Hg.) 2011: Handbuch des Bodenschutzes. Bodenökologie und -Belastung - Vorbeugende und abwehrende Schutzmaßnahmen 4. Weinheim: Wiley.

Burckhardt, Lucius (2013): Der kleinstmögliche Eingriff oder die Rückführung der Planung auf das Planbare. Berlin: Verlag Martin Schmitz.

Sausmikat, Klaus (Bauabteilungsleiter) 2016: Interview im Campusradio Kassel. Quelle: https://www.youtube.com/watch?v=QwVZudsZ6bQ (letzter Zugriff am 03.10.2016).

Schwarze, Michael (1987): Bürgerinitiativen - Widerstand und Selbsthilfe. In: Andritzky, Michael / Klaus Spitzer (Hg.), Grün in der Stadt: von oben, von selbst, für alle, von allen. Reinbek: Rowohlt, 398-420. 